EESTI NSV TEADUSTE AKADEEMIA TOIMETISED. FUOSIKA * MATEMAATIKA

ИЗВЕСТИЯ АКАДЕМИИ НАУК ЭСТОНСКОИ ССР. ФИЗИКА * МАТЕМАТИКА

PROCEEDINGS OF THE ACADEMY OF SCIENCES OF THE ESTONIAN SSR.

PHYSICS * MATHEMATICS

$1984,33,4$

P. TEHHO

удК 517.977 .5

\title{
СВОЙСТВА ДВУХШАГОВОГО ОПТИМАЛЬНОГО УПРАВЛЕНИЯ РЕГРЕССИОННОЙ МОДЕЛЬЮ СО СЛУЧАЙНЫМИ КОЭФФИЦИЕНТАМИ
}

R. TENNO. JUHUSLIKE KOEFITSIENTIDEGA REGRESSIOONVORRANDI KAHESAMMULISE OPTIMAALJUHTIMISE OMADUSI

R. TENNO. SOME PROPERTIES OF TWO STEP OPTIMAL CONTROL OF THE RANDOM COEFFICIENT REGRESSION EQUATIONS

(Представил Н. Алумяз)

Задачи дуального управления [ $\left.{ }^{1}\right]$ приходится решать во многих практических ситуациях, в связи с чем разработано немало приближенных алгоритмов этого управления $\left[{ }^{2,3}\right]$. Однако анализ свойств предложенных управлений проведен гораздо хуже. По-видимому, это связано с излишней общностью постановки задач. Нами поставлена цель исследовать сравнительно простую задачу дуального управления, имеющую обозримую структуру. При этом рассмотрены асимптотические свойства и достаточные условия единственности оптимального управления, найдена оценка сверху для разности стоимостей одношагового и оптимального управлений.

Задача управления. Предположим, что объект управления с выходом $x_{t}, t=1,2, \ldots, N$, и вектором входов (управлением) $u_{t-1}$ описывается регрессионной моделью

$$
x_{t}=u_{t-1}^{\mathrm{T}} \Theta_{t}+\xi_{t}
$$

со случайными коэффициентами $\Theta_{t}$. Помеха $\xi_{t}$ удовлетворяет уравнению

$$
\xi_{t}=\mu_{0}+\Phi\left(\xi_{t-1}-\mu_{0}\right)+\alpha_{t}, \quad \xi_{0}=\xi,
$$

где $\mu_{0}$, Ф - скалярные параметры, $\left(\alpha_{t}\right)-$ «белый шум», $\xi-$ (случайное) начальное условие. Измеряется

$$
y_{t}=x_{t}+h_{t}
$$

Пусть при каждом $t$ величины $h_{t}, \alpha_{t}, \Theta_{t}$, $\xi$ независимы и распределены по нормальному закону со средними

$$
M h_{t}=0, \quad M \alpha_{t}=0, \quad M \Theta_{t}=\mu_{1}, \quad M \xi=b
$$

и (ко-) вариациями

$$
\operatorname{var} h_{t}=r, \quad \operatorname{var} \alpha_{t}=\sigma, \quad \operatorname{cov} \Theta_{t}=D_{1}, \quad \operatorname{var} \xi=p,
$$

причем $r>0$, а матрица $D_{1}$ положительно определена. Требуется при за- 
данныт $\mu_{0}, \Phi, r, \sigma, \mu_{1}, D_{1}, b, p$ выбрать управление $u \equiv u_{0}$ на первом шаге такое, которое минимизирует двухшаговый $(N=2)$ функционал

$$
J=M\left\{\left(x_{1}-x^{0}\right)^{2}+\left(x_{2}-x^{0}\right)^{2}\right\},
$$

где $x^{0}$ - задающее воздействие, в предположении, что управление $u_{1}\left(y_{1}\right)$ на втором шаге выбрано оптимальным. Согласно алгоритму динамического программирования оптимальнос управлсние $u^{*}$ на первом шаге выбирается из условия минимума функции

$$
V(u)=S(u)+R(u),
$$

где $S(u)$ - риск действия, $R(u)$ - риск изучения. В нашем случае [ $\left.{ }^{4}\right]$

$$
\begin{aligned}
& S(u)=\left(a+u^{\mathrm{T}} \mu_{1}-x^{0}\right)^{2}+G+u^{\mathrm{T}} D_{1} u, \\
& R(u)=A-B T^{2},
\end{aligned}
$$

где

$$
\begin{aligned}
& A=K+\left(c-x^{0}\right)^{2} /\left(1+\mu_{1}^{\mathrm{T}} D_{1}^{-1} \mu_{1}\right), \quad B=\mu_{1}^{\mathrm{\top}} D_{1}^{-1} \mu_{1} /\left(1+\mu_{1}^{\mathrm{\top}} D_{1}^{-1} \mu_{1}\right), \\
& T^{2}=(\Phi G)^{2} /\left(r+G+u^{\mathrm{T}} D_{1} u\right),
\end{aligned}
$$

$a, c$ - оценки (прогнозы) возмущений $\xi_{1}, \xi_{2}$ (определенные до момента поступления наблюдений); $G, K$ - ковариации оценок $a, c$.

Одношаговым управлением $u^{+}$назовем такое управление, которое минимизирует функцию $S(u)$.

Асимптотические свойства оптимального управления. Рассмотрим в каком случае оптимальное $u^{*}$ и одношаговое управление $u^{+}$имеют сходные асимптотические свойства в том смысле, что при стремлении в бесконечность некоторого параметра е можно считать, что процесс изучения объекта происходит одинаково при любом управлении.

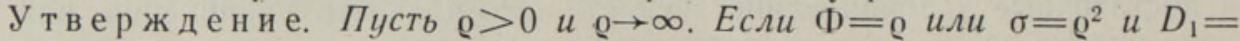
$=\mathrm{Q}^{2} L\left(\right.$ где $L-$ положительно определенная матрица), то $u_{\rho}{ }^{*} \rightarrow 0$, если $r=\mathrm{Q}^{2}$, то $u_{\mathrm{\rho}}{ }^{*} \rightarrow u^{+}$.

Д ок а з ат ел в с т о. Пусть $Ф=\varrho$. Тогда первые и вторые производные от функции $S(u), R(u)$ можно представить следующим образом:

$$
S_{u}=\varrho \tilde{S}_{1}(u, \varrho), \quad R_{u}=\varrho^{2} \widetilde{R}_{1}(u, \varrho), \quad R_{u u}=\varrho^{2} \widetilde{R}_{2}(u, \varrho),
$$

где $S_{1}(u, \cdot), \tilde{R}_{1}(\cdot, \cdot)$ - векторы, $R_{2}(\cdot, \cdot)$ - матрица, координаты (элементы) которых ограниченные функции. Из условия локального минимума функции $V(u)$ получим

$$
\varrho^{-1} \widetilde{S}_{1}(u, \varrho)+\widetilde{R}_{1}(u, \varrho)=0, \quad \varrho^{-2} S_{u u}+\widetilde{R}_{2}(u, \varrho)>0 .
$$

Условиям

$$
\lim _{\rho \rightarrow \infty} \widetilde{R}_{1}(u, \varrho)=2 B D_{1} u=0, \quad \lim _{\rho \rightarrow \infty} \widetilde{R}_{2}(u, \varrho)=2 B D_{1}>0
$$

удовлетворяет управление $\bar{u}=0$. Так как функция $S_{1}(u, \cdot)$ ограничена, а матрица $S_{u u}$ положительно определена, то из (3) видно, что $u_{\rho}^{*} \rightarrow 0$.

В случае $\sigma=\varrho^{2}$ и $D_{1}=\varrho^{2} L$ утверждение доказывается аналогично. В случае $r=Q^{2}$ вместо (3) получим

$$
S_{u}+\varrho^{-6} \tilde{R}_{1}(u, \varrho)=0, \quad S_{u u}+\varrho^{-4} \tilde{R}_{2}(u, \varrho)>0 .
$$

Условиям $S_{u}=0, S_{u u}>0$ удовлетворяет одношаговое управление $u^{+}$, функции $\widetilde{R}_{1}(\cdot, \cdot), \widetilde{R}_{2}(\cdot, \cdot)$ ограничены, и поэтому из (4) следует, что $u_{\rho}^{*} \rightarrow u^{+}$. 
3 а меч ан ие. Если $\Phi=0$, тоे асимптотические свойства оптимального и одношагового управлений отличаются, так как $\left|u_{\rho}{ }^{+}\right| \rightarrow \infty$, при $\varrho \rightarrow \infty$. Если $D_{1}=Q^{2} L$, то имеем $u_{\rho}{ }^{+} \rightarrow 0$.

Единственность оптимального управления. В случае одномерного управления можно получить следующее легко проверяемое условие единственности.

Неравенство

$$
(\Phi \Xi)^{2} B<4,
$$

где

$\Xi=G /(r+G)$, гарантирует единственность оптимального управления. Убедимся в этом. Строго выпуклая функция имеет максимум в единственной точке. Если для каждого управления функция $S_{u u}+R_{u и}$ положительна, то $V(u)$ строго выпукла. Поскольку $R_{u u}(u)$ снизу ограничена

$$
\min _{u} R_{u u}(u)=-0,5(\Phi \Xi)^{2} B D_{1},
$$

то требуемое выше условие выполняется, если функция

$$
S_{u u}+\min R_{u u}=2\left\{\mu_{1}^{2}+\left[1-0,25(\Phi \Xi)^{2} B\right] D_{1}\right\}
$$

$u$

положительная или если выражение в квадратных скобках положительное, что и является достаточным условием единственности оптимального управления.

Отметим, что если процесс аддитивных возмущений $\left(\xi_{t}\right)$ стационарный, то оптимальное управление единственное. Это следует из условий стационарности $|\Phi|<1$ и из того, что $B<1, \Xi<1$.

Оценка разности стоимостей одношагового и оптимального управлений. Ниже показано, что если потери от неоптимального управления большие, то это менее существенно связано с риском изучения, чем с риском действия. Поясним это более подробно.

Пусть $u^{+}-u=\delta$. Тогда а) из (1) видно, что

$$
S(u)-S\left(u^{+}\right)=\delta^{\mathrm{T}} M \delta \text {, }
$$

где

$$
M=\mu_{1} \mu_{1}^{\mathrm{T}}+D_{1}
$$

б) существует постоянная $N \geqslant 0$ такая, что для любого управления

$$
\left|R(u)-R\left(u^{+}\right)\right| \leqslant N|\delta| \text {. }
$$

В случае «больших» $|\delta|$ оценку (6) можно заменить более точной

$$
\left|R(u)-R\left(u^{+}\right)\right| \leqslant K, \quad K>0 .
$$

Докажем второе утверждение. По теореме о конечных приращениях в интервале $\left(u, u^{+}\right)$существует вектор $v$ такой, что

$$
R(u)-R\left(u^{+}\right)=R_{u}^{\mathrm{T}}(v) \delta .
$$

Следовательно, достаточно показать, что

$$
\left|R_{u}(v)\right| \leqslant N .
$$

После несложных преобразований, получим

$$
N=2 B\left[\Phi G /\left(r+G+\tilde{u}^{\mathrm{T}} D_{1} \tilde{u}\right)\right]^{2}\left|D_{1} \tilde{u}\right|,
$$

где $\tilde{u}-$ решение уравнения 


$$
\left(r+G+u^{\mathrm{T}} D_{1} u\right) D_{1}=4 D_{1} u u^{\mathrm{T}} D_{1} .
$$

Так как матрица $D_{1}$ положительно определена, то все решения уравнения (7) ограничены. Следовательно, длина вектора $D_{1} \widetilde{u}$ также является ограниченной.

Если $|\delta|$ — «большой» и $R\left(u^{+}\right) \geqslant R(u)$, то из определения (2) получим

$$
\begin{aligned}
& R\left(u^{+}\right)-R(u) \leqslant B \max _{u}\left[T^{2}(u)-T^{2}(u+\delta)\right] \leqslant \\
& \leqslant B\left[\max _{u} T^{2}(u)-\min _{u} T^{2}(u+\delta)\right]=B \frac{(\Phi G)^{2}}{r+G} .
\end{aligned}
$$

Если $R(u) \geqslant R\left(u^{+}\right)$, то получим также

$$
R(u)-R\left(u^{+}\right)<B \frac{(\Phi G)^{2}}{r+G} .
$$

Определим оценку разности стоимостей одношагового и оптимального управлений. Пусть $\delta=u^{+}-u^{*}$. Если $|\delta|-$ «алый», то из утверждений а), б) и определения $V(u)$ следует, что

$$
V\left(u^{+}\right)-V\left(u^{*}\right)=R\left(u^{+}\right)-R\left(u^{*}\right)-\delta^{\mathrm{T}} M \delta<N|\delta| .
$$

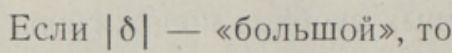

$$
V\left(u^{+}\right)-V\left(u^{*}\right) \leqslant K .
$$

Таким образом, потери оптимальности от одношагового управления ограничены сверху, причем малые потери определяются главным образом риском изучения.

Автор выражает благодарность В. Ольману за полезные замечания.

\section{ЛИ Т Е Р А Т У Р A}

1. Фельдбаум A. А. Основы теории оптимальных автоматических систем (2-е изд.). М., «Наука», 1966.

2. Asher, R. B., Andrisani II D., Donato, P. Proc IEEE, 64, 1226-1240 (1976).

3. Tse, E., Bar-Shalom, Y. Proc. of the 18th IEEE Conf. on Decision and Control Including the Symposium on Adaptive Processes, Fort Lauderdale Fla., 1979, 1, New York, 1979, 183-196.

4. Тенно Р. Изв. АН ЭССР. Физ. Матем., 32, № 1, 1-16 (1983).

Институт кибернетики

Академии наук Эстонской ССР
Поступила в редакцию 7/VI 1983

После переработки 23/IV 1984 